\title{
Efficacy and safety of cognitive enhancers for patients with mild cognitive impairment: a systematic review and meta-analysis
}

\author{
Andrea C. Tricco PhD MSc, Charlene Soobiah BSc, Shirra Berliner RN MSc, Joanne M. Ho MD, \\ Carmen H. Ng MSc BSc, Huda M. Ashoor BSc, Maggie H. Chen PhD MSc, Brenda Hemmelgarn MD PhD, \\ Sharon E. Straus MD MSC
}

\begin{abstract}
Background: Cognitive enhancers, including cholinesterase inhibitors and memantine, are used to treat dementia, but their effectiveness for mild cognitive impairment is unclear. We conducted a systematic review to examine the efficacy and safety of cognitive enhancers for mild cognitive impairment.
\end{abstract}

Methods: Our eligibility criteria were studies of the effects of donepezil, rivastigmine, galantamine or memantine on mild cognitive impairment reporting cognition, function, behaviour, global status, and mortality or harms. We identified relevant material by searching electronic databases (e.g., MEDLINE, Embase), the references of included studies, trial registries and conference proceedings, and by contacting experts. Two reviewers independently screened the results of the literature search, abstracted data and appraised risk of bias using the Cochrane risk-of-bias tool.

Results: We screened 15554 titles and abstracts and 1384 full-text articles. Eight ran- domized clinical trials and 3 companion reports met our inclusion criteria. We found no significant effects of cognitive enhancers on cognition (Mini-Mental State Examination: 3 randomized clinical trials [RCTs], mean difference [MD] 0.14, 95\% confidence interval [CI] -0.22 to 0.50; Alzheimer's Disease Assessment Scale - cognition subscale: 3 RCTs, standardized MD $-0.07,95 \% \mathrm{Cl}-0.16$ to 0.01$])$ or function (Alzheimer's Disease Cooperative Study activities of daily living inventory: 2 RCTs, MD $0.30,95 \% \mathrm{Cl}-0.26$ to 0.86 ). Cognitive enhancers were associated with higher risks of nausea, diarrhea and vomiting than placebo.

Interpretation: Cognitive enhancers did not improve cognition or function among patients with mild cognitive impairment and were associated with a greater risk of gastrointestinal harms. Our findings do not support the use of cognitive enhancers for mild cognitive impairment.
Competing interests:

Andrea Tricco,

Shirra Berliner, Joanne Ho,

Huda Ashoor, Maggie

Chen, Brenda Hemmelgarn and Sharon Straus have received grants from the Canadian Institutes for Health Research. Sharon Straus is an associate editor for CMAJ. She was not involved in the editorial decision-making process for this article. No other competing interests were declared.

This article has been peer reviewed.

Correspondence to:

Sharon E. Straus,

sharon.straus@utoronto.ca

CMAJ 2013. DOI: 10.1503 /cmaj.130451
$\mathrm{O}$ lder adults experiencing memory and cognition deficits without substantial limitations in activities of daily living may be given a diagnosis of mild cognitive impairment. ${ }^{1}$ These patients often present with subjective memory loss, impairment of cognitive function and no change in their basic daily functioning. Mild cognitive impairment has recently been recognized as a distinct condition, with a prevalence that ranges from $3 \%$ to $42 \%$ and increases with age. ${ }^{2}$ Because of the growing proportion of older adults worldwide, the prevalence of this condition will only increase in the future. ${ }^{3}$ Each year, 3\%-17\% of people with mild cognitive impairment experience progression to dementia, ${ }^{4-6}$ a rate that increases to between $11 \%$ and $33 \%$ by 2 years after the initial diagnosis. ${ }^{7}$ More than 4.6 million new cases of dementia are diagnosed each year, ${ }^{3}$ and efforts to reduce this public health burden are essential. Strategies to delay the progression of mild cognitive impairment are being sought to meet this challenge.

One strategy that has been hypothesized to delay the progression from mild cognitive impairment to dementia is the use of cognitive enhancers, agents that are often used to treat dementia. These medications include cholinesterase inhibitors (e.g., donepezil, rivastigmine and galantamine) and the $N$-methyl-D-aspartic acid receptor antagonist memantine. ${ }^{8}$ Donepezil, rivastigmine and galantamine increase the concentration of acetylcholine at neurotransmitter sites, ${ }^{9}$ enhancing the brain's cholinergic function. Galantamine also influences activity at nicotinic receptors, ${ }^{9}$ whereas memantine modulates the neurotransmitter glutamate. ${ }^{9}$ 
In many countries, cognitive enhancers are not widely available for patients with mild cognitive impairment. However, in some countries, including Canada, these drugs can be obtained through special authorization, ${ }^{10}$ and patients and their families are increasingly requesting their use. Although a Cochrane review on this topic exists, ${ }^{11}$ it does not provide information on the use of memantine for mild cognitive impairment or provide data on function or global status, nor does it distinguish between overall harms and treatment-related harms. We sought to examine the efficacy and safety of cognitive enhancers for mild cognitive impairment.

\section{Methods}

We followed the Preferred Reporting Items for Systematic Reviews and Meta-Analysis statement for reporting this review. ${ }^{12}$

We compiled a systematic review protocol based on guidance from the Preferred Reporting Items for Systematic reviews and Meta-Analysis for Protocols. ${ }^{13}$ We revised the protocol after receiving feedback from methodologists, pharmacy experts and clinicians. We published the final systematic review protocol in an open-access jour$\mathrm{nal}^{14}$ and registered our study with PROSPERO, the international prospective register of systematic reviews (registration no. CRD42012002234). Because the full methods for this study have been published previously, ${ }^{14}$ they are described only briefly here.

\section{Literature search}

We included studies involving patients with a diagnosis of mild cognitive impairment who were given one of 4 widely available cognitive enhancers (donepezil, rivastigmine, galantamine, memantine), which were compared with other cognitive enhancers, placebo or supportive care. Only experimental (e.g., randomized clinical trials [RCTs], quasi-RCTs, non-RCTs), quasi-experimental (e.g., interrupted time series, controlled before-and-after study) or observational epidemiology (e.g., cohort study) designs reporting cognition, function, behaviour, global status, and mortality or harms were included. All languages of dissemination, years of publication and types of articles (both published and unpublished) were eligible for inclusion.

We searched the following electronic databases: MEDLINE, Embase, the Cochrane Methodology Register, the Cochrane Central Register of Controlled Trials, the Cumulative Index of Nursing and Allied Health Literature, and Ageline. We supplemented our database search by searching trial registry websites, websites of organizations that produce guidelines and abstracts from conference proceedings. In addition, we contacted drug manufacturers. Finally, we scanned the reference lists of included studies and relevant reviews and contacted researchers and health care providers prolific in this area of research.

We conducted peer review of the MEDLINE literature search according to the Peer Review of Electronic Search Strategies checklist. ${ }^{15}$ After revising the search strategy in consultation with the research team, an experienced librarian conducted the literature searches on Nov. 23, 2011. The final MEDLINE search has been published previously, ${ }^{14}$ and the other search strategies are available from the authors upon request.

After a team training exercise, each citation (title and abstract) generated by the literature search were screened by 2 authors (ACT, CS, SB, $\mathrm{JMH}, \mathrm{CHN}$ ) independently using our preestablished eligibility criteria. Conflicts were resolved by discussion or the involvement of a third reviewer. The same process was followed to screen potentially relevant full-text articles, with the exception that, for some studies, the authors were contacted to determine study eligibility.

\section{Data abstraction}

We abstracted data for study characteristics including study design, the year in which the study was conducted and setting. We also abstracted data on patient characteristics, such as number of patients in the study, mean age, diagnostic criteria for mild cognitive impairment and comorbidities. To select the most relevant outcomes for data abstraction, we conducted an online survey with a convenience sample of 32 relevant policy-makers and clinicians known to provide care for patients with mild cognitive impairment. ${ }^{16}$ On the basis of the results of this exercise, we chose the following outcomes: cognition (measured by Alzheimer's Disease Assessment Scale - cognition subscale ${ }^{17}$ or MiniMental State Examination ${ }^{18}$ ), function (Alzheimer's Disease Cooperative Study activities of daily living inventory ${ }^{19}$ ), behaviour (Neuropsychiatric Inventory ${ }^{20}$ ), global status (Clinician's Interview-Based Impression of Change plus Caregiver ${ }^{21}$ ), overall and treatment-related mortality, and overall and treatment-related harms (e.g., nausea, vomiting, diarrhea, falls, headache, bradycardia and severe adverse events).

After a training exercise, each of the included studies was abstracted, independently, by 2 team members (ACT, CS, SB, JMH, CHN, HMA). Conflicts were resolved by discussion or the involvement of a third reviewer. Data were abstracted for 6, 12 and 24 weeks of follow-up, 
as well as the longest duration of follow-up. Major publications were sorted from companion reports (or duplicate publications), and study authors were contacted for data clarifications.

\section{Quality assessment}

The Cochrane risk-of-bias tool ${ }^{22}$ was used to appraise the risk of bias of the included RCTs. To examine the reporting of adverse drug reactions in the included studies, we used the McHarm tool. ${ }^{23}$ After calibrating the tools within the team, each of the included studies was appraised by 2 team members (CS, SB, JMH, CHN, HMA) independently. Conflicts were resolved by discussion or the involvement of a third reviewer.

\section{Data synthesis}

We synthesized the studies descriptively, with a summary of study characteristics, patient characteristics and the results of appraisals of methodological quality and risk of bias. We then pooled the studies using random-effects meta-analysis techniques in SAS version 9.2. ${ }^{24}$ We scrutinized 3 types of heterogeneity: clinical (e.g., type of patient population, diagnostic criteria, drug's mechanism of action), methodological (e.g., study duration) and statistical (e.g., $I^{2}$ statistic ${ }^{25}$ ). We examined important variables (e.g., time for assessment of outcomes, drug's mechanism of action) using meta-regression analysis $;{ }^{26}$ we con- ducted subgroup analysis if the meta-regression analysis was statistically significant.

\section{Results}

\section{Literature search}

The literature search yielded a total of 15554 titles and abstracts (Figure 1). Of these, we obtained 1384 full-text articles that were deemed potentially relevant for inclusion. Ten articles fulfilled our eligibility criteria, including 7 primary publications ${ }^{27-33}$ and 3 companion reports ${ }^{34-36}$ reporting on a total of $8 \mathrm{RCTs}$.

\section{Study characteristics}

All of the included studies were RCTs conducted between 1999 and 2007 in North America, Europe, New Zealand, Australia, South America, Israel and Turkey (Table 1). One of the RCTs did not report the study period, ${ }^{33}$ and 3 of the studies were multicentre trials. ${ }^{27,33}$ Four of the RCTs examined donepezil, ${ }^{28-31} 1$ study examined rivastigmine, ${ }^{32} 2$ studies (reported in a single publication) examined galantamine, ${ }^{27}$ and 1 study examined memantine. ${ }^{33}$ All included trials compared the cognitive enhancer with placebo. The number of patients in each study ranged from 51 to 1058 . The outcomes examined included the results of the Mini-Mental State Examination (3 studies), Alzheimer's Disease Assessment Scale - cognition subscale (5 studies), the

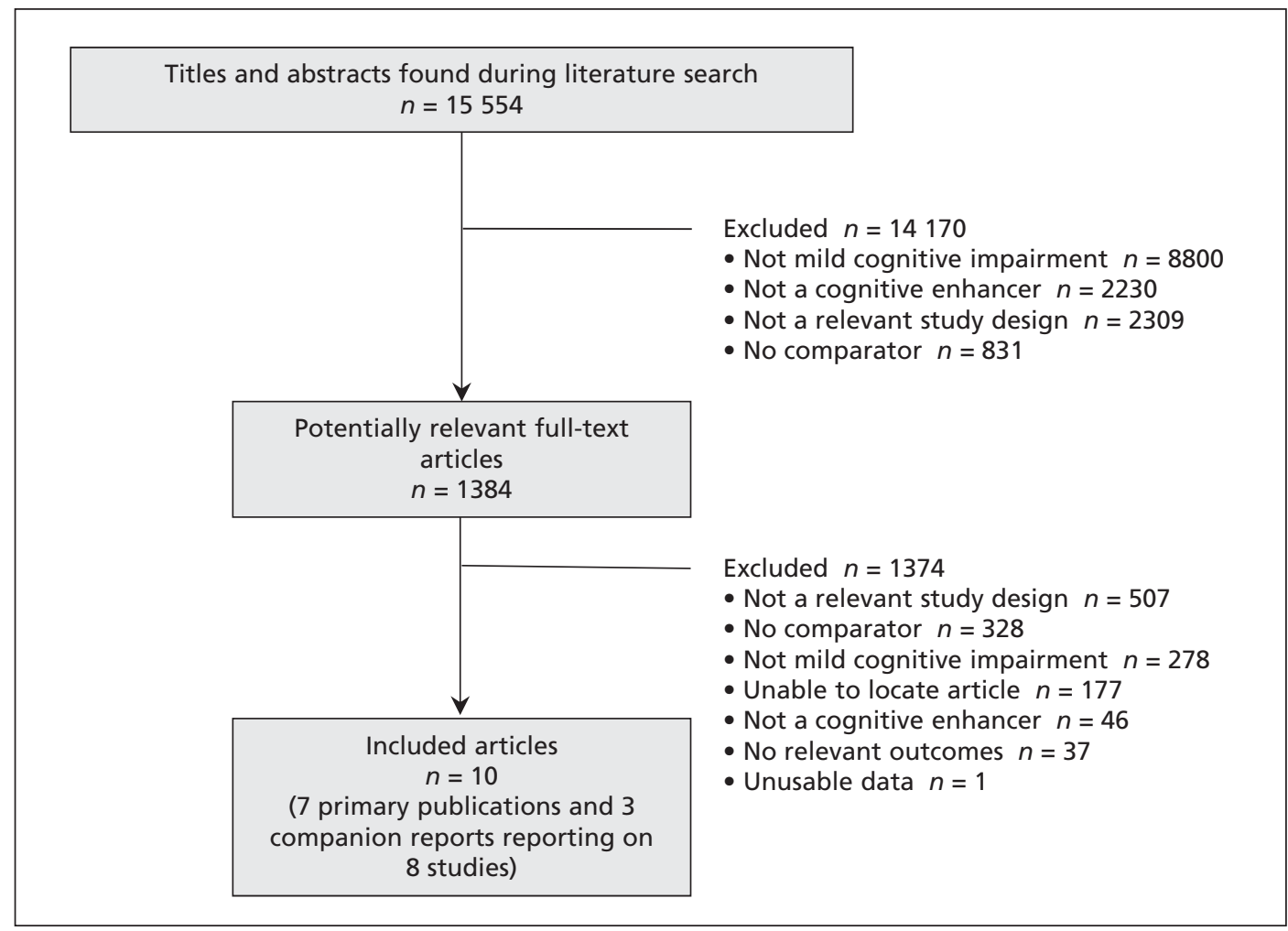

Figure 1: Identification of eligible studies. 
Alzheimer's Disease Cooperative Study activities of daily living inventory (2 studies) and the Neuropsychiatric Inventory (1 study), as well as mortality (3 studies) and harms (6 studies). In the publication that reported data on 2 separate trials, the harms were combined across the trials. ${ }^{27}$ None of the included studies reported the Clinician's
Interview-Based Impression of Change plus Caregiver outcome for assessing global status.

The percentage of women included in the RCTs ranged from $42.4 \%$ to $65.0 \%$, and the mean age of all patients ranged from about 66 to 73 years (Appendix 1, available at www.cmaj.ca /lookup/suppl/doi:10.1503/cmaj.130451/-/DC1).

Table 1: Characteristics of the included studies

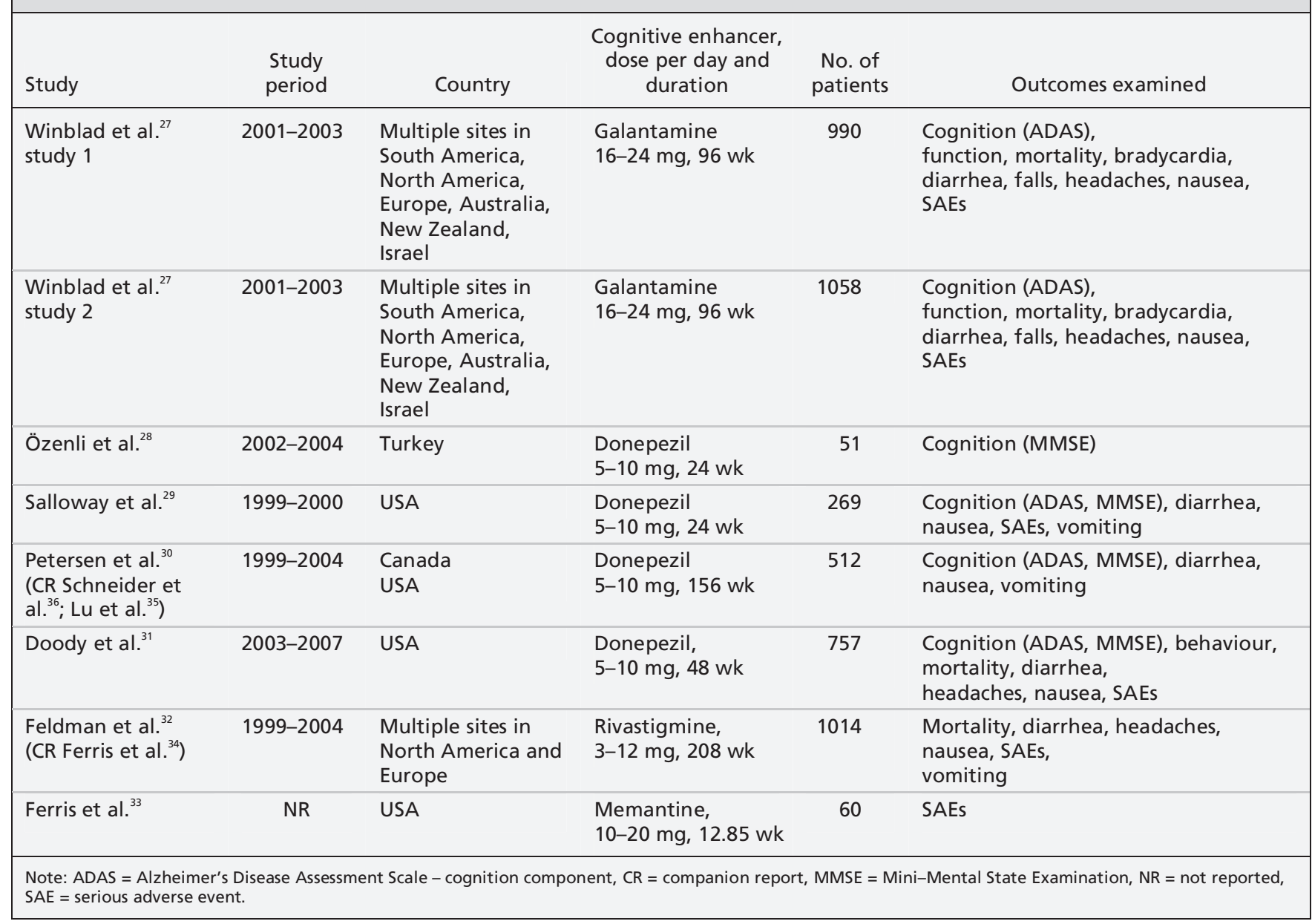

Table 2: Appraisal of risk of bias of the included studies using the Cochrane risk-of-bias tool ${ }^{22}$

\begin{tabular}{|c|c|c|c|c|c|c|c|}
\hline \multirow[b]{2}{*}{ Study } & \multicolumn{7}{|c|}{ Risk of bias } \\
\hline & $\begin{array}{c}\text { Random } \\
\text { sequence } \\
\text { generation }\end{array}$ & $\begin{array}{l}\text { Allocation } \\
\text { concealment }\end{array}$ & $\begin{array}{l}\text { Blinding of } \\
\text { patients, } \\
\text { personnel }\end{array}$ & $\begin{array}{l}\text { Blinding of } \\
\text { outcome } \\
\text { assessors }\end{array}$ & $\begin{array}{c}\text { Incomplete } \\
\text { outcome } \\
\text { data }\end{array}$ & $\begin{array}{l}\text { Selective } \\
\text { outcome } \\
\text { reporting }\end{array}$ & Other \\
\hline Winblad et al. ${ }^{27}$ study 1 & Unclear & Low & Low & Low & Low & Low & Low \\
\hline Winblad et al. ${ }^{27}$ study 2 & Unclear & Low & Low & Low & Low & Low & Low \\
\hline Salloway et al. ${ }^{29}$ & Unclear & Low & Low & Low & Unclear & Unclear & Unclear \\
\hline Petersen et al. ${ }^{30}$ & Unclear & Unclear & Unclear & Unclear & Low & Unclear & Low \\
\hline Doody et al. ${ }^{31}$ & Low & Low & Low & Low & Low & Unclear & Unclear \\
\hline Feldman et al. ${ }^{32}$ & Low & Low & Low & Low & Low & Low & Unclear \\
\hline Ferris et al. ${ }^{33}$ & Unclear & Unclear & Low & Unclear & High & Unclear & Unclear \\
\hline
\end{tabular}


Most of the studies used the Clinical Dementia Rating to diagnose mild cognitive impairment with a cut-off of $0.5^{27,30-32}$ or a range of $0.5-1.0 .^{29}$ The Mini-Mental State Examination was also used across the studies to identify cognitive deficits or to exclude dementia, with a cut-off ranging from 24 to $30,{ }^{30,31,33}$ the inclusion of patients with scores of at least 24 but no higher than $26^{28}$ or patients with a score of at least $24 .^{29}$

\section{Study quality}

Using the Cochrane risk-of-bias tool, ${ }^{22} 3$ studies $^{27,32}$ were appraised as having a low risk of bias on 6 of 7 items (Table 2). One study was deemed to have a high risk of bias for 1 item, ${ }^{33}$ and the rest of the studies had an unclear risk of bias for at least 2 criteria.

The 6 RCTs that included harms as study outcomes did not adequately report these outcomes
(Appendix 2, available at www.cmaj.ca/lookup /suppl/doi:10.1503/cmaj.130451/-/DC1). Seven of the 14 criteria on the McHarm tool were poorly reported by most of the included trials (Appendix 2).

\section{Clinical outcomes}

After a median of 36 weeks of follow-up, there was no difference in cognition, as measured by the Mini-Mental State Examination, between patients who received donepezil and those who received placebo (3 RCTs, mean difference [MD] $0.14,95 \%$ confidence interval [CI] -0.22 to $0.50, I^{2}=0 \%$; Figure 2). However, we saw a trend toward favouring donepezil. Metaregression analysis showed no differences in cognition for a variety of follow-up periods ranging from 24 to 156 weeks.

Similarly, we saw no significant difference in

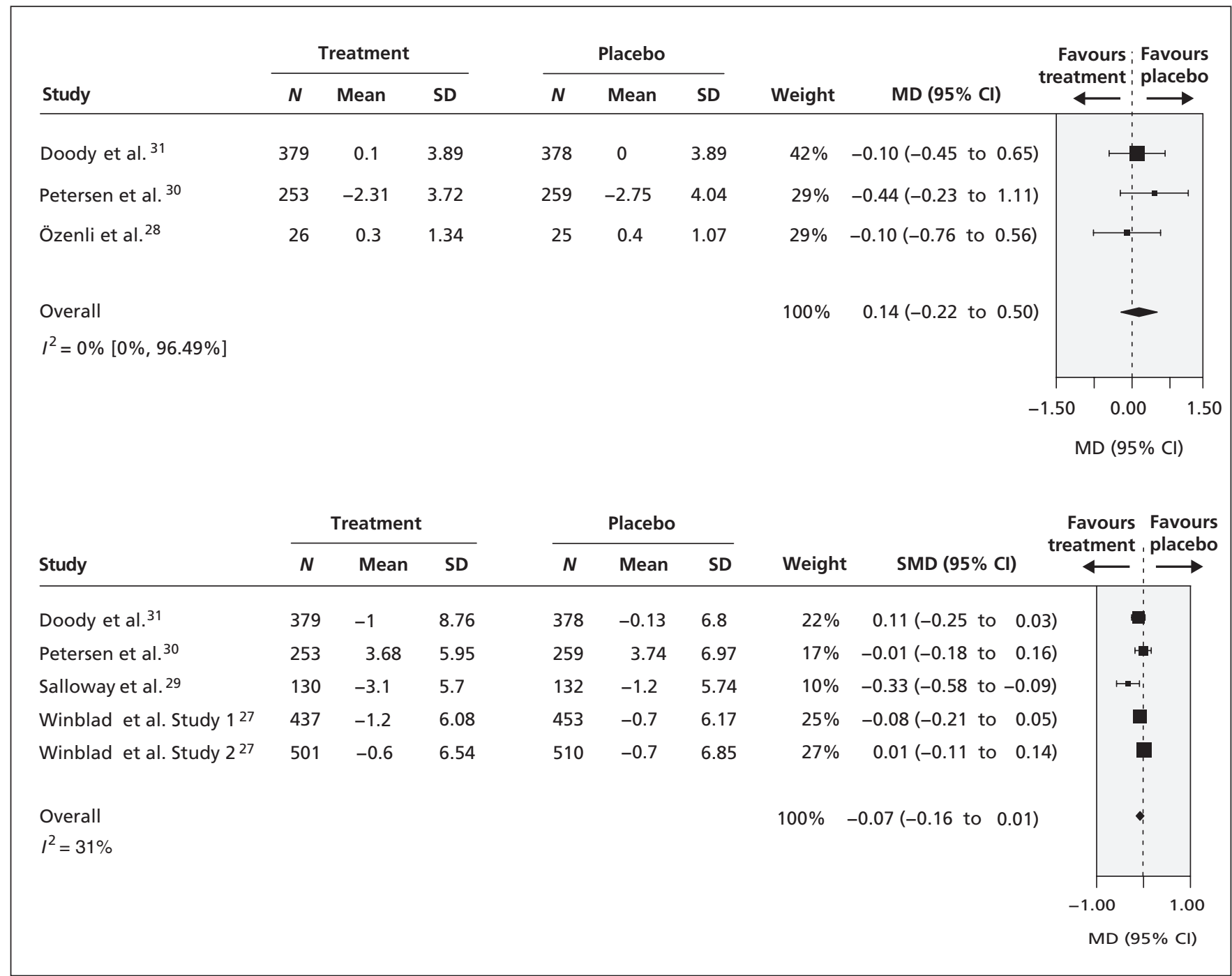

Figure 2: Comparison of effects of donepezil (5-10 mg/d) and placebo on patient cognition as assessed by the Mini-Mental State Examination after a median follow-up of 36 weeks and by the Alzheimer's Disease Assessment Scale - cognition component after a median follow-up of 24 weeks. Standardized MD (SMD) was calculated by standardizing the results of the studies to a uniform scale using the study effect relative to the standard deviation (SD) in each study. ${ }^{37} \mathrm{Cl}=$ confidence interval, $\mathrm{MD}=$ mean difference. 
cognition, as measured by the Alzheimer's Disease Assessment Scale - cognition component, between donepezil or galantamine and placebo after a median of 24 weeks of follow-up (5 RCTs, standardized MD $-0.07,95 \% \mathrm{CI}-0.16$ to $0.10, I^{2}=31 \%$; Figure 2). In addition, we found no significant differences with this form of $\operatorname{cog}$ nitive assessment for drugs with different modes of action (i.e., donepezil v. galantamine). However, we did see a significant trend for follow-up periods ranging from 6 to 156 weeks in the meta-regression analysis. Specifically, the results were statistically significant in favour of cognitive enhancers over placebo for studies providing data after 12-84 weeks of follow-up (including 1531 patients), but not for studies providing data after 85-96 weeks of follow-up (including 1901 patients).

After 96 weeks of follow-up, there was no significant difference in functional status, as measured by the Alzheimer's Disease Cooperative Study activities of daily living inventory, between patients who received galantamine and those who received placebo (2 RCTs, MD 0.30, 95\% CI 0.26 to $0.86, I^{2}=0 \%$; Figure 3 ), although we saw a trend toward favouring placebo.

In the single RCT that reported behavioural outcomes as assessed by the Neuropsychiatric Inventory, ${ }^{31}$ there was no significant difference between patients who received donepezil $(5-10 \mathrm{mg} / \mathrm{d})$ and those who received placebo after 48 weeks of follow-up (MD 0.8, 95\% CI -0.59 to 2.19 ).

There was no significant difference in overall mortality between patients who received a cognitive enhancer (donepezil, rivastigmine or galantamine) and those who received placebo after a median of 156 weeks of follow-up (3 RCTs, relative risk [RR] $1.84,95 \%$ CI 0.41 to $8.20, I^{2}=$
$80 \%$; Figure 4), although a trend toward favouring placebo was seen. Meta-regression and subgroup analyses showed no significant differences in mortality between agents with different modes of action (i.e., donepezil and rivastigmine v. galantamine), and these analyses were associated with less statistical heterogeneity. Only 1 RCT reported treatment-related mortality, with no significant differences between donepezil and placebo (RR 2.97, 95\% CI 0.31 to 28.4). ${ }^{31}$

The frequency of nausea and diarrhea were significantly greater among patients taking cognitive enhancers (donepezil, rivastigmine or galantamine) than among those taking placebo after a median of 126 weeks of follow-up (nausea: 4 RCTs, RR $3.04,95 \%$ CI 2.52 to $3.66, I^{2}=$ 21\%; diarrhea: 4 RCTs, RR $2.33,95 \%$ CI 1.74 to $3.13, I^{2}=55 \%$, Appendix 3, available at www .cmaj.ca/lookup/suppl/doi:10.1503/cmaj.130451 /-/DC1). The results were similar for vomiting (donepezil or rivastigmine, median 208 weeks of follow-up; 3 RCTs, RR 4.40, 95\% CI 3.21 to $6.03, I^{2}=0 \%$ ) (Appendix 3) and headaches (rivastigmine or galantamine, median 152 weeks of follow-up; 2 RCTs, RR $1.27,95 \%$ CI 1.04 to $1.53, I^{2}=18 \%$ ) (Appendix 3). However, we saw no significant differences in serious adverse events between cognitive enhancers (donepezil, rivastigmine, galantamine or memantine) and placebo after a median of 48 weeks of follow-up (4 RCTs, RR $0.97,95 \%$ CI 0.86 to $1.10, I^{2}=$ $0.0 \%$ ) (Appendix 3). Notably, only 1 study explicitly defined serious adverse events, as "any untoward medical occurrence which resulted in death, was life threatening, required or prolonged inpatient hospitalization, or resulted in persistent or significant disability/incapacity." ${ }_{31}$ Meta-regression and subgroup analyses showed no significant differences between agents with

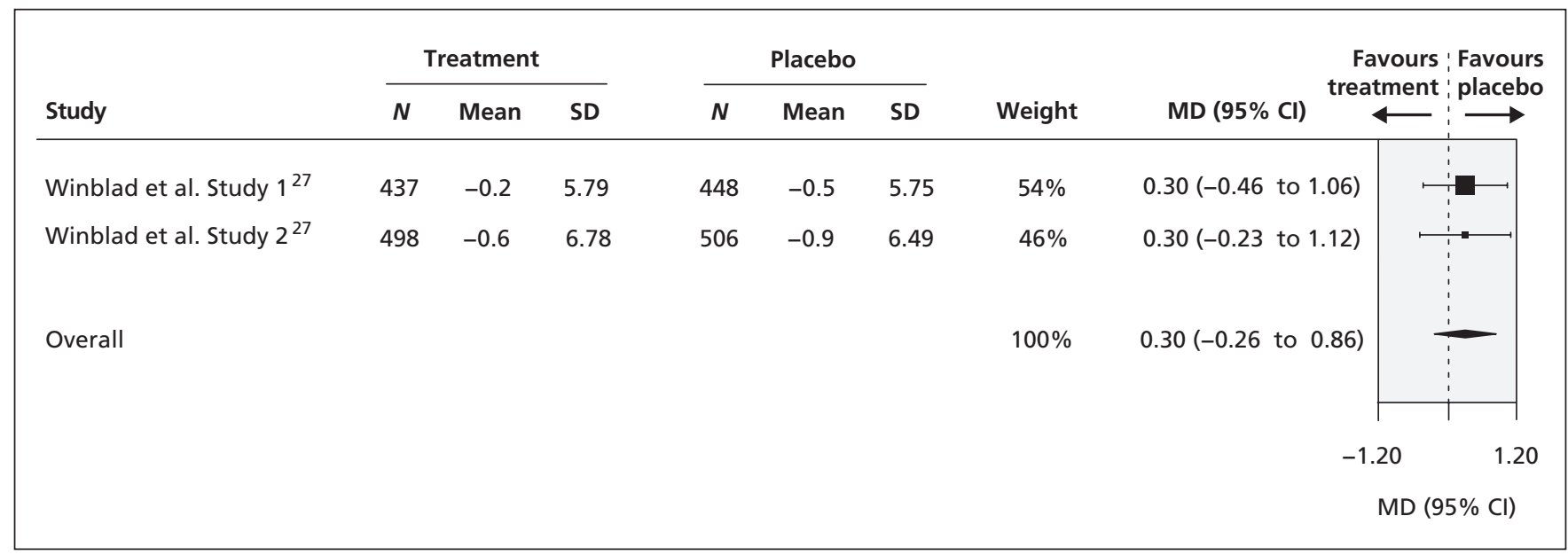

Figure 3: Comparison of effect of galantamine (16-24 mg/d) and placebo on patients' functional status as assessed by the Alzheimer's Disease Cooperative Study activities of daily living inventory after 96 weeks of follow-up. $\mathrm{Cl}=$ confidence interval, $\mathrm{MD}=\mathrm{mean}$ difference, SD = standard deviation. 
different modes of action across all of the harm outcomes, and these analyses were associated with less statistical heterogeneity.

One study reported treatment-related harms. ${ }^{31}$ After 48 weeks of follow-up, significantly more of the patients taking donepezil had nausea (RR 2.21, 95\% CI 1.27 to 3.11), diarrhea (RR 4.87, 95\% CI 2.73 to 8.70 ) and headache (RR 2.23, 95\% CI 1.14 to 4.33 ) relative to patients who received placebo. ${ }^{31}$

Another study, which compared galantamine and placebo, reported the proportion of patients who experienced falls and bradycardia after 96 weeks follow-up. ${ }^{27}$ Significantly more patients receiving galantamine experienced bradycardia (RR $1.52,95 \%$ CI 1.04 to 2.22 ), yet significantly fewer patients receiving the drug experienced falls (RR $0.71,95 \%$ CI 0.52 to 0.98 ).

\section{Interpretation}

We found statistically significant results for cognitive enhancers compared with placebo based on the results of the Alzheimer's Disease Assessment Scale - cognition component after 12-84 weeks of follow-up, but this difference was absent beyond that point. This finding suggests that, to ensure accuracy of results, trialists should conduct longer-term RCTs in this area. A statistically significant trend favouring cognitive enhancers was seen based on results of the Mini-Mental State Examination; however, the effect size was not clinically important. None of the other outcomes showed statistical signifi- cance, suggesting that these agents do not slow the progression to dementia.

The results of our meta-analysis showed that patients taking these medications experienced significantly more nausea, diarrhea, vomiting and headaches than patients taking placebo. Furthermore, patients taking these medications might have been at greater cardiac risk, with 1 study finding a higher incidence of bradycardia among patients who received galantamine. ${ }^{27}$ Patients and their families should consider this information when requesting these medications. Similarly, health care decision-makers may not wish to approve the use of these medications for mild cognitive impairment, because these drugs might not be effective and are likely associated with harm.

Our results are similar to those of 2 Cochrane reviews examining the use of cognitive enhancers for mild cognitive impairment. ${ }^{11,38} \mathrm{Nei}-$ ther of those reviews found any significant improvements in cognition, and both reviews found significantly more harms associated with these agents. The most recent Cochrane review included 9 studies, ${ }^{11} 3$ of which were excluded from our review because they included mixed patient populations with vascular dementia ${ }^{39,40}$ or did not include our outcomes of interest. ${ }^{41}$ Conversely, we included 3 studies that were not included in the recent Cochrane review.

\section{Limitations}

When planning this review, we hoped to identify pragmatic trials, quasi-experimental studies and

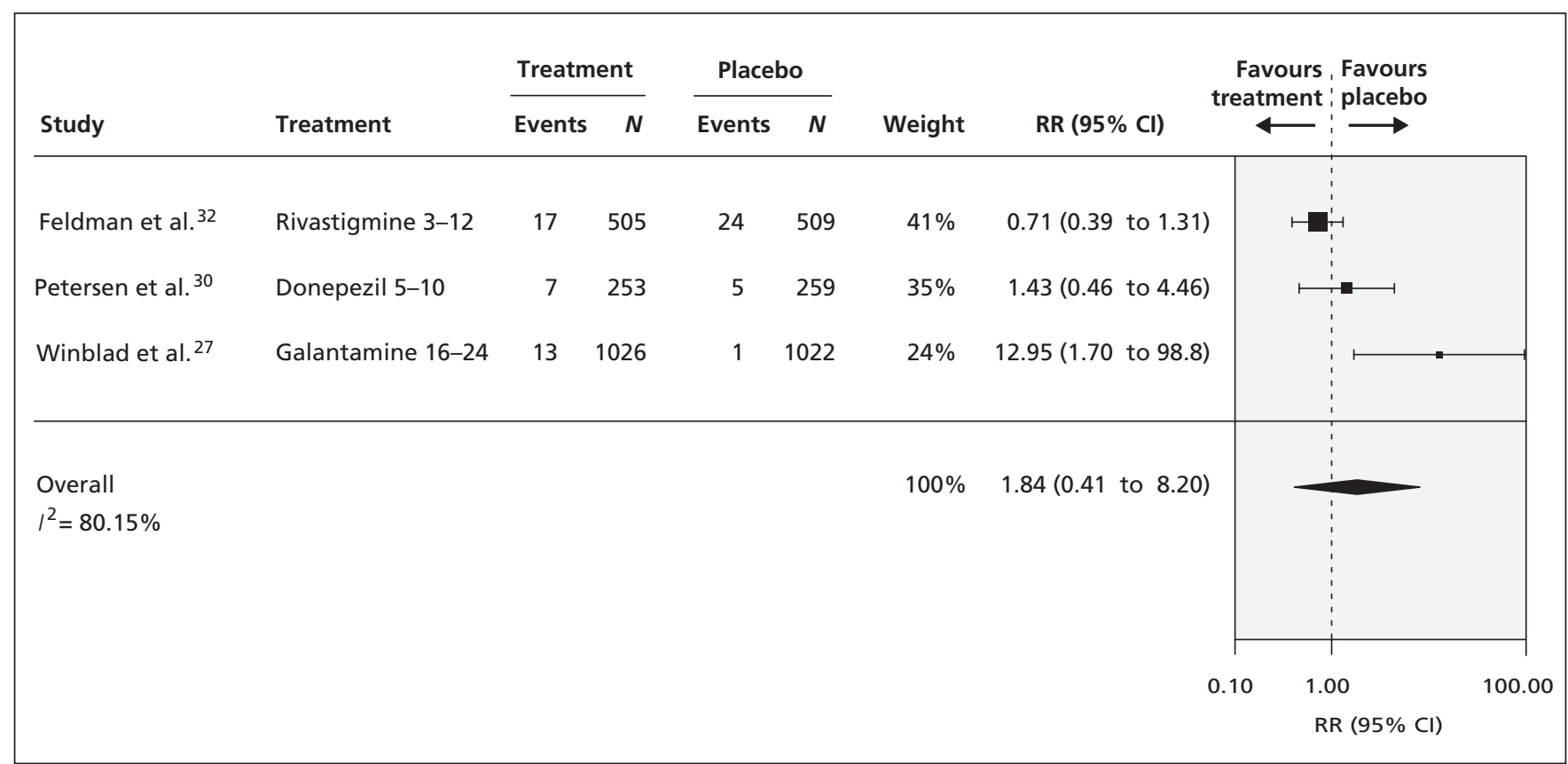

Figure 4: Comparison of effect of cognitive enhancers and placebo on overall mortality after a median follow-up of 156 weeks. $\mathrm{Cl}=$ confidence interval, $\mathbf{R R}=$ relative risk. 
cohort studies that would allow us to determine the comparative effectiveness and safety of cognitive enhancers for mild cognitive impairment. However, our extensive literature search identified no such studies. Furthermore, we had originally planned to conduct a network metaanalysis but were unable to do so because of the dearth of literature in this area. Network metaanalysis allows comparison of treatments that have not been compared directly in head-to-head trials $^{42}$ and can be used to rank interventions in terms of effectiveness and safety. ${ }^{42}$ Updates of this systematic review should attempt to conduct such an analysis.

Most of the studies included in our metaanalysis did not report comorbidities, which makes generalization difficult, the definition of mild cognitive impairment was inconsistent across studies, and many of the studies employed short follow-up periods. Furthermore, half had a rating of "unclear" for at least 3 of the 7 Cochrane risk-of-bias criteria, and most had inadequate reporting for at least 7 of the $14 \mathrm{McHarm}$ criteria. This finding likely reflects poor reporting of the included trials, and it is recommended that trialists in this area use the Consolidated Standards of Reporting Trials ${ }^{43}$ in future research.

Although we searched extensively for unpublished studies, we identified none for inclusion, which suggests that our results might be influenced by publication bias. However, the dearth of eligible studies prevented us from formally assessing the impact of publication bias using a funnel plot or regression analysis. ${ }^{44}$ Furthermore, we had to exclude some potentially relevant studies because they did not report our outcomes of interest or provide the data in a usable format. We were unable to conduct subgroup analysis by risk of bias owing to the small number of studies included. Finally, our choice of outcomes for the study was based on a survey of a convenience sample of clinicians and policy-makers, and a different group might have chosen a different set of outcome measures. However, the outcomes examined here are common in the dementia literature, particularly in research about Alzheimer dementia. ${ }^{16}$ Moreover, as mentioned previously, the clinicians in our sample were actively seeing patients with mild cognitive impairment in practice and thus were in a good position to judge the usefulness of various outcome tools.

\section{Conclusion}

Our results do not support the use of cognitive enhancers for patients with mild cognitive impairment. These agents were not associated with any benefit and led to an increase in harms. A decision aid should be developed that would allow patients to properly weigh the benefits and disadvantages of taking these medications in countries where they are available for this indication.

\section{References}

1. Chertkow H. Diagnosis and treatment of dementia: introduction. Introducing a series based on the Third Canadian Consensus Conference on the Diagnosis and Treatment of Dementia. CMAJ 2008; 178:316-21.

2. Ward A, Arrighi HM, Michels S, et al. Mild cognitive impairment: disparity of incidence and prevalence estimates. Alzheimers Dement 2012;8:14-21.

3. Ferri CP, Prince M, Brayne C, et al. Global prevalence of dementia: a Delphi consensus study. Lancet 2005;366:2112-7.

4. Farias ST, Mungas D, Reed BR, et al. Progression of mild cognitive impairment to dementia in clinic- vs community-based cohorts. Arch Neurol 2009;66:1151-7.

5. Busse A, Angermeyer MC, Riedel-Heller SG. Progression of mild cognitive impairment to dementia: a challenge to current thinking. Br J Psychiatry 2006;189:399-404.

6. Landau SM, Harvey D, Madison CM, et al. Comparing predictors of conversion and decline in mild cognitive impairment. Neurology 2010;75:230-8.

7. Ritchie K. Mild cognitive impairment: an epidemiological perspective. Dialogues Clin Neurosci 2004;6:401-8.

8. Dementia: a NICE-SCIE guideline on supporting people with dementia and their carers in health and social care. Leicester (UK): British Psychological Society and London (ON): The Royal College of Psychiatrists; 2007.

9. Perras C, Shukla VK, Lessard C, et al. Cholinesterase inhibitors for Alzheimer's disease: a systematic review of randomized controlled trials [Technology report no. 58]. Ottawa (ON): Canadian Coordinating Office for Health Technology Assessment; 2005.

10. Pedone C, Lapane KL, Mor V, et al. Donepezil use in US nursing homes. Aging Clin Exp Res 2004;16:60-7.

11. Russ TC, Morling JR. Cholinesterase inhibitors for mild cognitive impairment. Cochrane Database Syst Rev 2012;9:CD009132.

12. Moher D, Liberati A, Tetzlaff J, et al. Preferred reporting items for systematic reviews and meta-analyses: the PRISMA statement. BMJ 2009;339:b2535.

13. Moher D, Shamseer L, Clarke M, et al. Reporting guidelines for systematic review protocols. Proceedings of the 19th Cochrane Colloquium; 2011 Oct. 19-22; Madrid (Spain).

14. Tricco AC, Soobiah C, Lillie E, et al. Use of cognitive enhancers for mild cognitive impairment: protocol for a systematic review and network meta-analysis. Syst Rev 2012;1:25.

15. Sampson M, McGowan J, Cogo E, et al. An evidence-based practice guideline for the peer review of electronic search strategies. J Clin Epidemiol 2009;62:944-52.

16. Soobiah C, Straus SE, Blondal E, et al. Outcomes utilized to assess Alzheimer's disease from a systematic review and survey of decision-makers. Proceedings from the Canadian Geriatrics Society 33rd Annual Scientific Meeting; 2012 Apr. 18-20 Toronto (ON).

17. Rosen WG, Mohs RC, Davis KL. A new rating scale for Alzheimer's disease. Am J Psychiatry 1984;141:1356-64.

18. Folstein MF, Folstein SE, McHugh PR. "Mini-mental state." A practical method for grading the cognitive state of patients for the clinician. J Psychiatr Res 1975;12:189-98.

19. Galasko D, Bennett D, Sano M, et al. An inventory to assess activities of daily living for clinical trials in Alzheimer's disease. The Alzheimer's Disease Cooperative Study. Alzheimer Dis Assoc Disord 1997;11(Suppl 2):S33-9.

20. Cummings JL, Mega M, Gray K, et al. The neuropsychiatric inventory: comprehensive assessment of psychopathology in dementia. Neurology 1994;44:2308-14.

21. Knopman DS, Knapp MJ, Gracon SI, et al. The Clinician Interview-Based Impression (CIBI): a clinician's global change rating scale in Alzheimer's disease. Neurology 1994;44:2315-21.

22. Higgins JP, Altman DG, Gotzsche PC, et al. The Cochrane Collaboration's tool for assessing risk of bias in randomised trials. BMJ 2011;343:d5928.

23. Santaguida P, Raina P. The development of the McHarm Quality Assessment Scale for adverse events. 2008. Available: http://hiru.mcmaster.ca/epc/mcharm.pdf (accessed 2013 Sep. 3).

24. DerSimonian R, Laird N. Meta-analysis in clinical trials. Control Clin Trials 1986;7:177-88.

25. Higgins JP, Thompson SG. Quantifying heterogeneity in a metaanalysis. Stat Med 2002;21:1539-58.

26. van Houwelingen HC, Arends LR, Stijnen T. Advanced methods in meta-analysis: multivariate approach and meta-regression. Stat Med 2002;21:589-624. 
27. Winblad B, Gauthier S, Scinto L, et al. Safety and efficacy of galantamine in subjects with mild cognitive impairment. Neurology 2008;70:2024-35.

28. Özenli Y, Yagci D, Karaca S. Efficacy of donepezil on cognitive functions in mild cognitive impairment. Klinik Psikofarmakoloji Bulteni 2007;17:62-7.

29. Salloway S, Ferris S, Kluger A, et al. Efficacy of donepezil in mild cognitive impairment: a randomized placebo-controlled trial. Neurology 2004;63:651-7.

30. Petersen RC, Thomas RG, Grundman M, et al. Vitamin E and donepezil for the treatment of mild cognitive impairment. N Engl J Med 2005;352:2379-88.

31. Doody RS, Ferris SH, Salloway S, et al. Donepezil treatment of patients with MCI a 48-week randomized, placebo-controlled trial. Neurology 2009;72:1555-61.

32. Feldman HH, Ferris S, Winblad B, et al. Effect of rivastigmine on delay to diagnosis of Alzheimer's disease from mild cognitive impairment: the InDDEx study. Lancet Neurol 2007;6:501-12.

33. Ferris S, Schneider L, Farmer M, et al. A double-blind, placebo-controlled trial of memantine in age-associated memory impairment (memantine in AAMI). Int J Geriatr Psychiatry 2007;22:448-55.

34. Ferris S, Nordberg A, Soininen H, et al. Progression from mild cognitive impairment to Alzheimer's disease: effects of sex, butyrylcholinesterase genotype, and rivastigmine treatment. Pharmacogenet Genomics 2009;19:635-46.

35. Lu PH, Edland SD, Teng E, et al. Donepezil delays progression to AD in MCI subjects with depressive symptoms. Neurology 2009;72:2115-21.

36. Schneider LS, Raman R, Schmitt FA, et al. Characteristics and performance of a modified version of the ADCS-CGIC CIBIC+ for mild cognitive impairment clinical trials. Alzheimer Dis Assoc Disord 2009;23:260-7.

37. Higgins J, Green S, editors. Cochrane handbook for systematic reviews of interventions. Hoboken (NJ): John Wiley \& Sons; 2009.

38. Birks J, Flicker L. Donepezil for mild cognitive impairment. Cochrane Database Syst Rev 2006; (3):CD006104.

39. Peters O, Lorenz D, Fesche A, et al. A combination of galantamine and memantine modifies cognitive function in subjects with amnestic MCI. J Nutr Health Aging 2012;16:544-8.

40. Narasimhalu $\mathrm{K}$, Effendy $\mathrm{S}$, Sim $\mathrm{CH}$, et al. A randomized controlled trial of rivastigmine in patients with cognitive impairment no dementia because of cerebrovascular disease. Acta Neurol Scand 2010;121:217-24.

41. Koontz J, Baskys A. Effects of galantamine on working memory and global functioning in patients with mild cognitive impairment: a double-blind placebocontrolled study. Am J Alzheimers Dis Other Demen 2005;20:295-302.

42. Lumley T. Network meta-analysis for indirect treatment comparisons. Stat Med 2002;21:2313-24.

43. Schulz KF, Altman DG, Moher D. CONSORT 2010 statement: updated guidelines for reporting parallel group randomised trials. PLoS Med 2010;7: e1000251.

44. Egger M, Davey Smith G, Schneider M, et al. Bias in meta-analysis detected by a simple, graphical test. BMJ 1997;315:629-34.

Affiliations: Li Ka Shing Knowledge Institute (Tricco, Soobiah, Berliner, Ho, Ng, Ashoor, Chen, Straus), St. Michael's Hospital, Toronto, Ont.; Departments of Medicine and Community Health Sciences (Hemmelgarn), University of Calgary, Calgary, Alta.; Department of Geriatric Medicine (Straus), University of Toronto, Toronto, Ont.

Contributors: Andrea Tricco helped conceive and design the study, collected and analyzed the data, interpreted the results and wrote the manuscript. Charlene Soobiah, Shirra Berliner, Joanne Ho, Carmen Ng and Huda Ashoor collected the data. Maggie Chen revised the manuscript for important intellectual content. Brenda Hemmelgarn helped conceive the study and revised the manuscript for important intellectual content. Sharon Straus helped conceive and design the study, interpreted the results and helped write the manuscript. All the authors approved the final version submitted for publication.

Funding: This systematic review was funded by the Drug Safety and Effectiveness Network/Canadian Institutes of Health Research (DSEN/CIHR; grant no. 257990). Andrea Tricco is funded by a DSEN/CIHR New Investigator Award in Knowledge Synthesis. Sharon Straus is funded by a Tier 1 Canada Research Chair in Knowledge Translation. The study sponsor had no role in the design of the study, the collection, analysis or interpretation of the data, the writing of the report or the decision to submit the article for publication.

Acknowledgments: The authors thank Dr. Sumit R. Majumdar, Dr. Muhammad Mamdani, Dr. Charles Goldsmith, Dr. David Juurlink and Dr. Joseph Beyene for their valuable feedback on the conceptualization of this review. The authors also thank Dr. Bin Dong for conducting some of the analyses and Dr. Jemila Hamid for updating the plots; Laure Perrier for conducting the literature search and screening some of the citations; Jennifer D'Souza for locating full-text articles; David Hallett and Erin Lillie for screening some of the citations; Marco Ghassemi and Erik Blondal for abstracting some of the data; and Lianne Kark Ezer and Dr. Paul Kahn for identifying some of the trial protocols and conference abstracts and for scanning reference lists; and Peggy Robinson for assistance with manuscript preparation. 